\title{
History and Advantages of Best Source Selection for Today's Modern Telemetry Applications Along With the Benefits of Different Approaches
}

\author{
Stephen Nicolo \\ GDP Space Systems \\ A Division of Delta Information Systems \\ 747 Dresher Road \\ Horsham, Pennsylvania 19044-2247 USA \\ Phone: (215) 657-5270 \\ snicolo@gdpspace.com
}

\begin{abstract}
:
Best Source Selection (BSS) has become a major topic at many modern telemetry ranges and ground stations around the world today. Best Source Selection techniques for telemetry applications, have been around for well over 25 years in one form or another. With the introduction of Next Generation Correlating Best Source Selection techniques a decade ago, the BSS function has evolved into an extremely powerful tool for the real-time and post flight recovery of telemetry data. These Next Generation techniques, currently operational at a variety of ranges around the world today, provide significant performance increases at these sites. As ranges continued to evolve, moving toward more network centric solutions, Correlating Best Source Selectors have also followed this trend while still dealing with the issues of encrypted data, latency and switching from stream-to-stream without dropping lock on downstream frame synchronizers.
\end{abstract}

This paper describes the evolution of Best Source Selection as well as the advantages and disadvantages of a variety of different approaches and BSS modes of operation that are available today.

Key words: Data Acquisition, Best Source Selection, Correlating Best Source Selectors, DQE

\section{What is a Best Source Selector?}

In the telemetry world there are many situations where there is a transmitting device sending telemetry information to several ground antenna receiving sites. In these types of applications, the transmitters are typically moving (i.e. aircraft, ground vehicle or missile). At any point in time the best received signal with the minimum amount of interference is changing as a result of noise, obstructions and / or signal reflections. These applications require an automatic and dynamic method of selecting the best source of data (best receiving site) at every point in time during the mission. One of many examples of this is a flight test application. In this application the aircraft under test continually transmits the test data to several receiving sites on the ground. These receiving sites are typically located at various points along the path of the test aircraft (refer to
Figure 1). In Figure 1, the transmitted signal from the aircraft (\#1) is received by three antennas (\# 2, $3 \& 4)$. The received signal is then routed through receivers and bit synchronizers to a Best Source Selector unit (\#5). The Best Source Selector (BSS) then selects the best signal for output (\#6). At any point in time, based on the position of the transmitter on the aircraft with respect to a particular receiving site, one or more receiving sites has a better signal than the others. In these applications, a system (Best Source Selector) that can quickly detect and react (i.e. switch to the best signal) is required so that the end result is Best Source Selector data output that is better than any individual input over time.

This document explores the evolution of Best Source Selection in the telemetry world. It also points out the pros and cons of a variety of different approaches and modes, and describes 
the many different modes that are available to solve this challenging problem as well as the significant performance gains that can be achieved with the latest FPGA technologies that are available today.

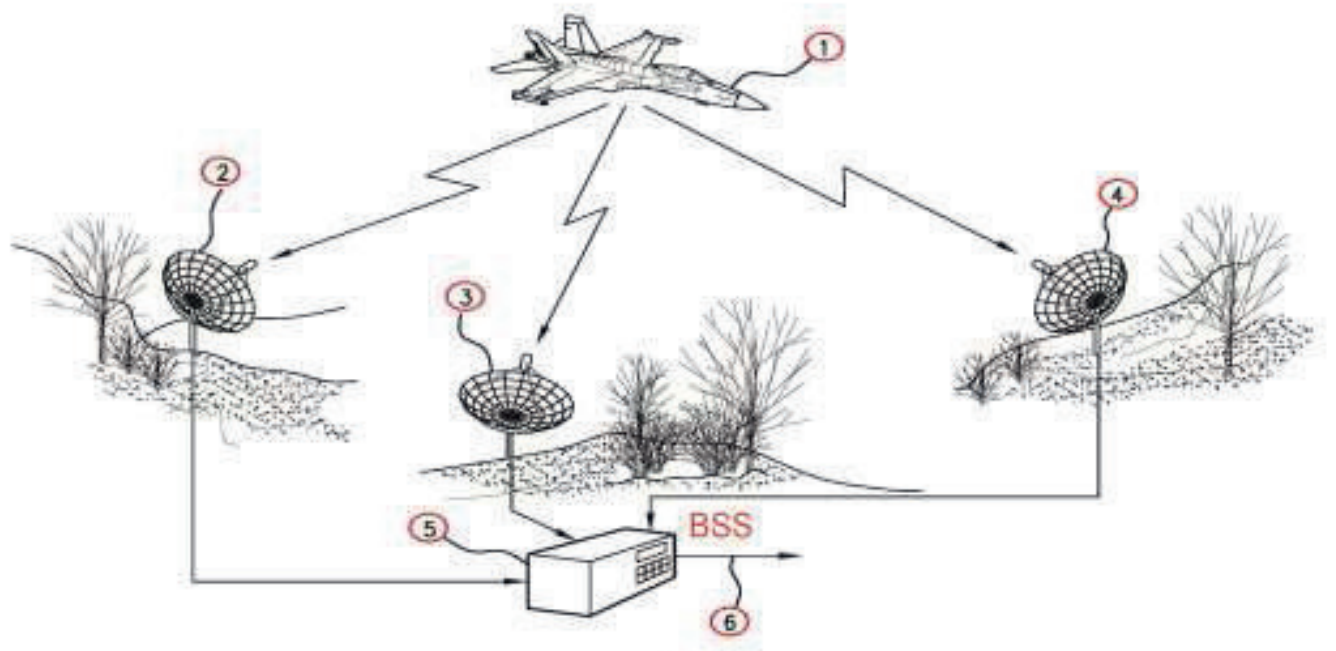

Figure 1. Example Flight Test Best Source Selector Application

\section{Definition of the Problem}

As streams are received from different sources they are routed to a central location for processing and Best Source Selection. Since time delays can vary from source to source, based on transmission distance and a variety of different equipment delays, data is skewed in both phase and time as it is received at the Best Source Selector. Probably, the most serious problem is that of data loss due to the switching process and / or noise bursts. These error bursts typically happen at the worst possible time (during the test maneuver), when critical data is required (i.e. aircraft / vehicle maneuver in the flight test example). Figure 2 depicts the misalignment of the streams as seen by the Best Source Selector. Because of these skews between streams, if the Best Source Selector does not handle this properly, the BSS switches from stream to stream resulting in time jumps and drop-locks on downstream frame synchronizers from the selected BSS output. This was one of the drivers in the evolution of Best Source Selectors toward correlating the streams in time to each other (Correlating Best Source Selectors). A second problem is the ability to do Best Source Selection of encrypted data.

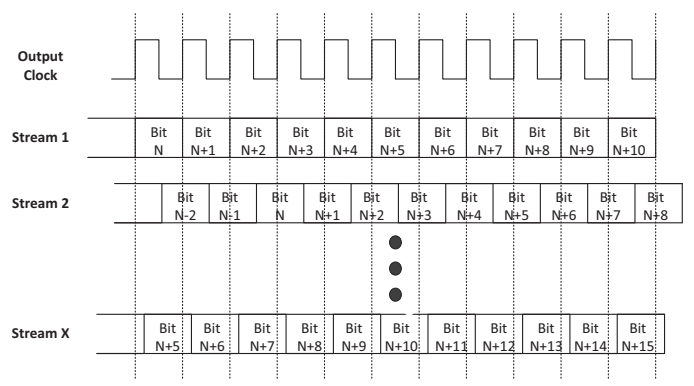

Figure 2 Raw Data (Not Phase or Time Aligned)

In Figure 2 the clock associated with the Best Source Stream is used as the Best Source output clock. In Figure 2 this happens to be Stream 1 at this point in time. Note however that any of the streams could have been the primary stream if it had been deemed to be the best stream based on quality metrics that are discussed further in this document. Notice that each rising edge of the output clock is lined up (synchronous) with the data transitions in Stream 1 (Stream 1 is in phase with the output clock). Since time delays can vary from source to source, based on transmission distance and a variety of different equipment delays, the phase and data content of the other streams are not lined up with the output clock (i.e. they do not switch synchronously with the rising edge of the output clock). Since in a Correlating Best Source Selector we regenerate the Best Source output bit stream on a bit-by-bit basis, all candidate streams must be 
synchronous and time aligned with the output clock. This is one of the key problems that a modern BSS had to overcome. Methods of dealing with this problem for both clear and encrypted data are discussed in further detail in the following sections of this document.

\section{History/Evolution of Best Source Selectors}

In the late 80's and 90's Best Source Selectors were basically frame synchronizers with a selector switch on the output. The user had to have some knowledge of the path of the aircraft for best results. Each stream in a group was assigned a priority based on the path of the mission. The Best Source Selector logic basically started with the highest priority stream that was in lock. When the current stream dropped lock, the Best Source Selector output would switch to the next highest priority stream that was in lock. This is illustrated in figure 3 below.

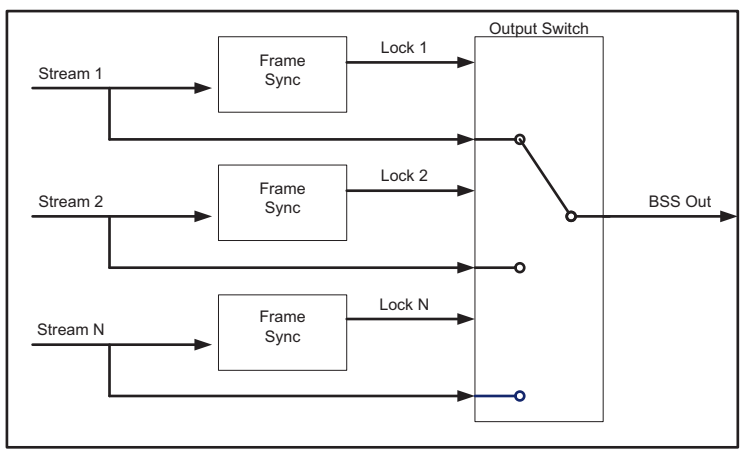

Figure 3 Non-Correlating Legacy Best Source Selector

Frame Sync lock was used as the indicator that the received stream was good or bad. As with most frame synchronizers, lock strategies (Allowable Search, Check, Lock \& Flywheel Errors) could be programmed for each stream allowing them to ride through short bursts of errors in the stream without dropping lock, which would cause the unit to switch to another stream. This is because in early Best Source Selectors the streams were not correlated in time to each other and frequent switching was not desirable. At switch time, jumps and loss of lock on downstream frame synchronizers would occur. Another issue with this approach was that the BSS could not handle encrypted data because a clear frame sync pattern was required for switch decisions.

In the 2000's, as higher density FPGAs became more prevalent and as PC processing power increased significantly in the industry, they were adapted to Best Source Selector applications. There was a major push to correlate the streams in time to each other in the Best Source Selector. This is because once the streams were correlated, there would be no interruption in the downstream data from the BSS output when the BSS switched from stream to stream. This made it acceptable to switch more often as the data quality of each of the streams dynamically changed. There was also a push for more complex modes of operation and algorithms for establishing quality of the streams for better performance of the BSS. With data stream correlation now possible, a next generation of Correlating Best Source Selectors was born.

For the correlation of the streams and the implementation of new BSS algorithms there are several different approaches from different vendors to accomplish this task. These approaches basically fell into two main categories: the PC Based Approach using PC hard drives and the hardware based FPGA approach using high density FPGAs, and solid state memories for the temporary storage and processing of BSS data. In the PC approach, frame synchronizers were again used to synchronize to the incoming streams and store these streams in buffers on the hard drive in the computer. The PC correlated the data streams in time to each other based on the framed data on the drive. This was again very processor intensive and although the PC could handle an individual stream at rates of up to the required $40 \mathrm{Mbps}$, there was a maximum total aggregate rate for all streams together (all streams could not simultaneously operate properly at maximum rate). In a 16 stream system example, there was a limit to how many streams could be processed based on rates and number of streams. This was because the $\mathrm{PC}$ would reach its processing limit. Like the other legacy frame synchronizer based units, these units could not handle encrypted data until the introduction of RCC Encapsulated streams in late 2017. The RCC DQE/DQM is discussed further later in this document.

In the high density FPGA based solution; a completely different approach was taken from day one. The FPGA introduced a new correlation method based on the data content itself, which is totally independent of the frame synchronizer pattern. In this approach, state machines continually matched patterns in the actual data itself to correlate the streams in time to each other. Once the streams were correlated, the state machines would continually check correlation every 256 bits. This high speed, OS independent, FPGA based approach allowed the Correlating Best Source Selector to handle correlation of all streams at maximum bit rate simultaneously with no maximum aggregate rate limit and no 
requirements for high speed $\mathrm{PCs}, \mathrm{PC}$ Operating Systems and hard drives. With this FPGA approach for correlation of data based on data content and not frame synchronizer pattern, this approach could handle encrypted data from the start. In fact, due to the randomized nature of encrypted data, the FPGA approach of correlating on data content worked better with encrypted data (it is totally unique and easier to correlate). This FPGA based approach was the first approach that was able to support encrypted streams and it was introduced in the 2008 time-frame.

Figure 4 illustrates the incoming candidate streams for Best Source Selection both phase aligned and time aligned. With this new phase and time alignment of the streams, lossless switching between streams was now possible opening the way for more complex algorithms and modes of operation. These modes and methods of BSS are discussed in more detail later in this document.

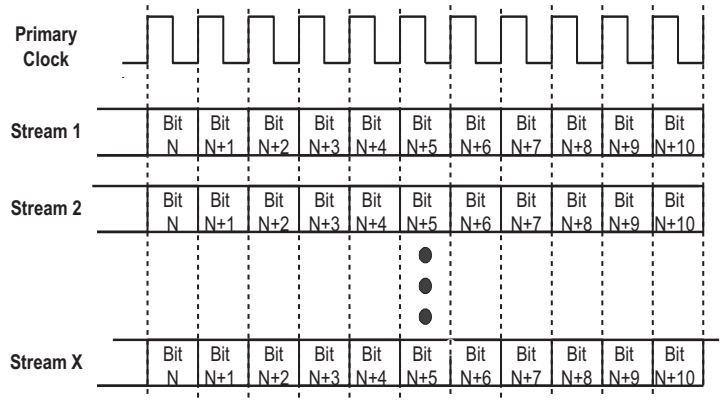

Figure 4 Streams Aligned in both Phase and Time Allowing Lossless Switching

\section{Data Transport}

Early Next Generation Correlating Best Source Selectors (CBSS) either used digital modes for data quality (quality based on errors in the frame sync pattern) or they used analog modes in which signal quality was assigned at the bit level (data quality for each bit) in bit synchronizers located in the Correlating Best Source Selector. These modes of operation are discussed in more detail later in this document. Around 2010 the bit synchronizers with Best Source Selector capability were not only available in the Correlating Best Source itself but were located at remote sites. At these remote sites bit synchronizers locked to the incoming data stream and assigned quality metrics at the remote site based on distortion of the received analog output of the receivers. Data, along with quality information, was then sent to the Correlating Best Source Selectors in a transfer frame and Data Quality Encapsulation (DQE) was born. This DQE transfer frame had a frame sync pattern along with data and interleaved quality information. This allowed more flexibility in the CBSS system implementation and allowed further separation of the antennas. With DQE, the antenna could be over 50 miles away and still used in the Correlating Best Source Selector (CBSS) mix. The data along with quality information from the remote site were sent back to the CBSS as data and clock. In recent years, DQE was moved from the stand-alone bit synchronizer to the bit synchronizer in the receiver. This allowed receiver and bit synchronizer parameters in the receiver to generate data quality information, which today is called Data Quality Metrics (DQM). Today there are still several different modes for DQE and DQM (both legacy bit-by-bit and the newer RCC type based on thousands of bits). They each have their application and their benefits. Better Correlating Best Source Selector units support all of the available BSS modes and additionally support mixed modes. The support of mixed modes is a very important feature. With mixed modes operators can use different modes at the same time for optimum performance in any application. Having several different modes in their toolbox allows the user the flexibility to handle any application. Modes are discussed further in the next section.

Early Data Quality Encapsulation (DQE) implementations output data and clock which was transported over a variety of transport media at ranges (i.e. Ethernet, Multiplexers, Microwave, etc.) from remote sites to the CBSS. More recently, several types of DQE/DQM are able to be output directly from receivers in both Data/Clock format and also Ethernet transport. Better CBSS units accept data along with quality from transport streams in both Data/Clock and also from Ethernet directly.

\section{Data Quality and Next Generation Correlating Best Source Selector Advanced Modes of Operation}

With the availability of new technologies (Larger FPGAs and Higher Speed Computers) along with the challenges from industry to provide better more powerful implementations of Best Source Selection, telemetry manufacturers came up with a variety of new modes and algorithms. These could be utilized along with the new types of Correlating Best Source Selectors that provided lossless switching and additional performance gains. The CBSS output stream could now perform several $d B$ better than the best input stream. Lossless switching (Correlating Best Source Selectors) was huge in the evolution of Best Source Selectors. These new advancements, which 
went along with Best Source Selection, included new methods for determining the quality of the stream. One vender (GDP) even determined quality down to the bit level for maximum performance in multipath situations. The data and the quality information was then stored into memory to be used by these new algorithms. The challenge was to do this in real time with minimal impact on latency.

The initial legacy systems used in the 90's depended on Frame Sync Lock for the determination of a good or bad stream. This was simple and straight forward. Progressions over the next two decades introduced a variety of different modes and algorithms. They include the initial digital modes and later analog modes along with a variety of different metrics all of which, in one way or another, come back to the error rate or error rate probability of the candidate streams for CBSSs. Several key modes utilized today are discussed in further detail in the following paragraphs.

\section{Digital Pattern Mode (Pattern Eb/No):}

In the Digital Pattern (Pattern Eb/No) mode, frame sync lock is still used in the Correlating Best Source Selector algorithm. In addition to frame sync lock, additional quality information is also gathered based on statistical errors in the frame sync pattern. This mode, by itself, can be used when legacy receiver and bit synchronizer hardware is being used that do not have the ability to provide the newer additional quality information to the Correlating Best Source Selector. It is not as fast to react as other modes because quality is determined on a small number of bits in each minor frame (frame sync pattern). Because of this, it takes many frames of data to calculate a useful quality measurement as a stream degrades or gets better. If three or more streams are available this mode can be used in conjunction with Majority Vote for several additional $\mathrm{dB}$ of performance gain (depending on the number of available streams). The switchover to Majority Vote should be quick and automatic as soon as three or more streams are available. This mode cannot be used when the stream is encrypted but is a very useful mode to have in your toolkit for situations when legacy hardware is being used that does not support some of the newer modes of best source selection.

\section{RCC Mode DQE/DQM Mode:}

In late 2017, the Range Commanders Council came up with a method to assign a Data Quality Metric (DQM), which was basically a Bit Error Probability (BEP) of each consecutive block of data from the incoming stream. This Data Quality Metric (16 bit quality value), which translated directly to Bit Error Probability (BEP) from a defined table, was assigned to a block of data to be used in best source selection. A 16 bit quality value for each block of data (the DQM) was included in a special Data Quality Encapsulation (DQE) transfer frame to get the data blocks from the receiver to the Correlating Best Source Selector. The header for each block was 48 bits. The header contained a 16 bit frame sync pattern, the 16 bit DQM value for that particular block of data and some other information bits. Although the block size is programmable (minimum $1 \mathrm{~K}$ bits / maximum $16 \mathrm{~K}$ bits), the size that was initially qualified and used was $4 \mathrm{~K}$ bits. The DQE transfer frame with data, allowed other BSS implementations that did not have the ability to measure quality of the analog baseband signals in their internal bit synchronizers, to now be able to handle encrypted data streams. This was because the quality information was passed to the BSS via the DQE stream. This is a solid mode of operation which is much better than the straight digital mode that looked for errors in the sync pattern. It could also be used in conjunction with a Majority Vote mode, when three or more streams are available, for performance gain on the BSS output. This mode has less overhead than the GDP bit-by-bit mode. However, with 2 streams there is no performance gain and it still has only 1 quality value for a very large block of data. Since this mode is fairly new, additional performance testing must still be done to further quantify this mode of operation. Further definition of this mode is provided in IRIG10617 Chapter 2, Appendix 2-G.

\section{CRC Mode:}

The cyclic redundancy check or CRC is a technique for detecting errors in received digital data. CRC information is a code word containing a fixed number of bits that is appended to a defined block of transmitted data bits. The CRC information is based upon the multiplication of a polynomial with the data contained in the data block. By processing the received data blocks with the same polynomial in the same way as the transmitting system, the receiving system is capable of determining if errors exist in the received data. If the block of data has no errors, it can be used in the CBSS output.

When added to a telemetry frame that is passed over a data link, the CRC may be used to determine the quality of the transmitted bitstream. The CRC word is appended as the last word contained in each minor frame of the telemetry stream produced by the transmitter. Pad bits (zeroes) may be added after the CRC, if needed, to permit decommutation of the 
frame in a fixed number of bits per word. Calculation of the CRC is based upon the data block beginning with the first bit of the Frame Synchronization Pattern and ending with the last bit of the word preceding the CRC word (the entire telemetry payload). Since it is possible for a highly errored frame to be reported by the CRC test as OK, bit errors found in the frame synchronization pattern are also measured.

When the data stream is received by the CBSS, an error check is performed based upon the received $\mathrm{CRC}$ word. The received $\mathrm{CRC}$ is compared with the calculated $\mathrm{CRC}$ at the receiver. If a $\mathrm{CRC}$ error is detected, a "flag" bit is set to indicate this situation. The error bit (flag) is used by the best source selector as a part of its decision making process. Since a single bit error will cause a bad CRC value (stream is in error) this mode should also be used in conjunction with other modes like Majority Vote. When all streams are bad and there are more than 3 streams a Majority Vote will be performed. CRC mode is not widely used, but it is another available tool in the CBSS toolkit.

\section{GDP Weighted Majority Vote /Analog Bit-by- bit Mode with GDP Data Quality Encapsulation (GDP DQE):}

This mode is the most powerful mode in the Correlating Best Source Selector toolset and it should be utilized in any situation where in can be used. It has been available and operational for well over 10 years now. It is unique because it provides a quality measurement for each bit. This mode of operation has three configurations. The configuration that is used depends on the application. The first implementation includes full-up bit synchronizers as part of the Correlating Best Source Selector (CBSS) unit itself. The bit synchronizer takes the raw analog video output from the receiver. From this raw analog output signal, quality is determined based on distortions in the analog signal. The second implementation is where the bit synchronizer is located external to the CBSS. In this mode, the legacy receiver is typically at a remote location. A bit synchronizer with Data Quality Encapsulation ability is placed at the remote site. It measures the quality of the analog signal from the receiver and sends the data along with interleaved quality information to the CBSS. The third more recent implementation is to send this information with the data directly out of the receiver (via data/clock and/or Ethernet). When data is received, quality of the stream is determined and the data along with interleaved quality information is sent to the Correlating Best Source Selector. Because this mode determines quality of the received analog signal it does not matter if the data is encrypted or clear.

In all cases there are two quality measurements made on the data. The first is a Long Term Signal Quality, measured over 256 bits. This is used in the validation of the stream and tells the CBSS the basic health of each received stream. The second measurement is a bit-by-bit Short Term Signal Quality (Quality of each bit). This information is then stored in the CBSS in solid state memories along with the data for each of the candidate streams. To achieve the full benefit of the Weighted Majority Vote mode, three or more data streams are needed. However, significant performance gain is still achieved with only two streams utilizing the bitby-bit signal quality information alone. This ability to get a performance gain (greater than $2 \mathrm{~dB}$ ) with 2 streams and the fact that the data can be encrypted is huge. With this mode enabled, when there are only two valid streams present, they are automatically correlated in time. The bit-by-bit output is then determined based on the bit-by-bit signal quality. Figure 5 illustrates, at a high level, how this algorithm works. For example: if one of the two streams has a signal quality which is $5 \mathrm{~dB}$ better than the other stream in a particular bit location, the bit value of the better stream is output in that bit location. Although error correction occurs in this mode, maximum error correction performance is achieved when three or more valid sources are present.

When a third source comes on-line, the FPGA state machines automatically correlate this stream with the other two sources and full majority voting (weighted by the short and long term Quality) is invoked. For example, with three sources, the corresponding bit location in each of the sources is examined and a vote occurs with the signal quality bits present to determine the validity of the data bits. This mode corrects the output data stream when error bursts occur due to receiver fades and / or multi-path interference. In this mode, a substantial link performance gain is achieved of better than three times the error rate squared. For example three sources with an error rate of $10^{-3}$ result in an output error rate better than $3 \mathrm{x}$ $10^{-6}$. This translates to performance gains of better than $2 \mathrm{~dB}$ for two streams, better than 3 $\mathrm{dB}$ for three streams and better than $5 \mathrm{~dB}$ for four streams. 


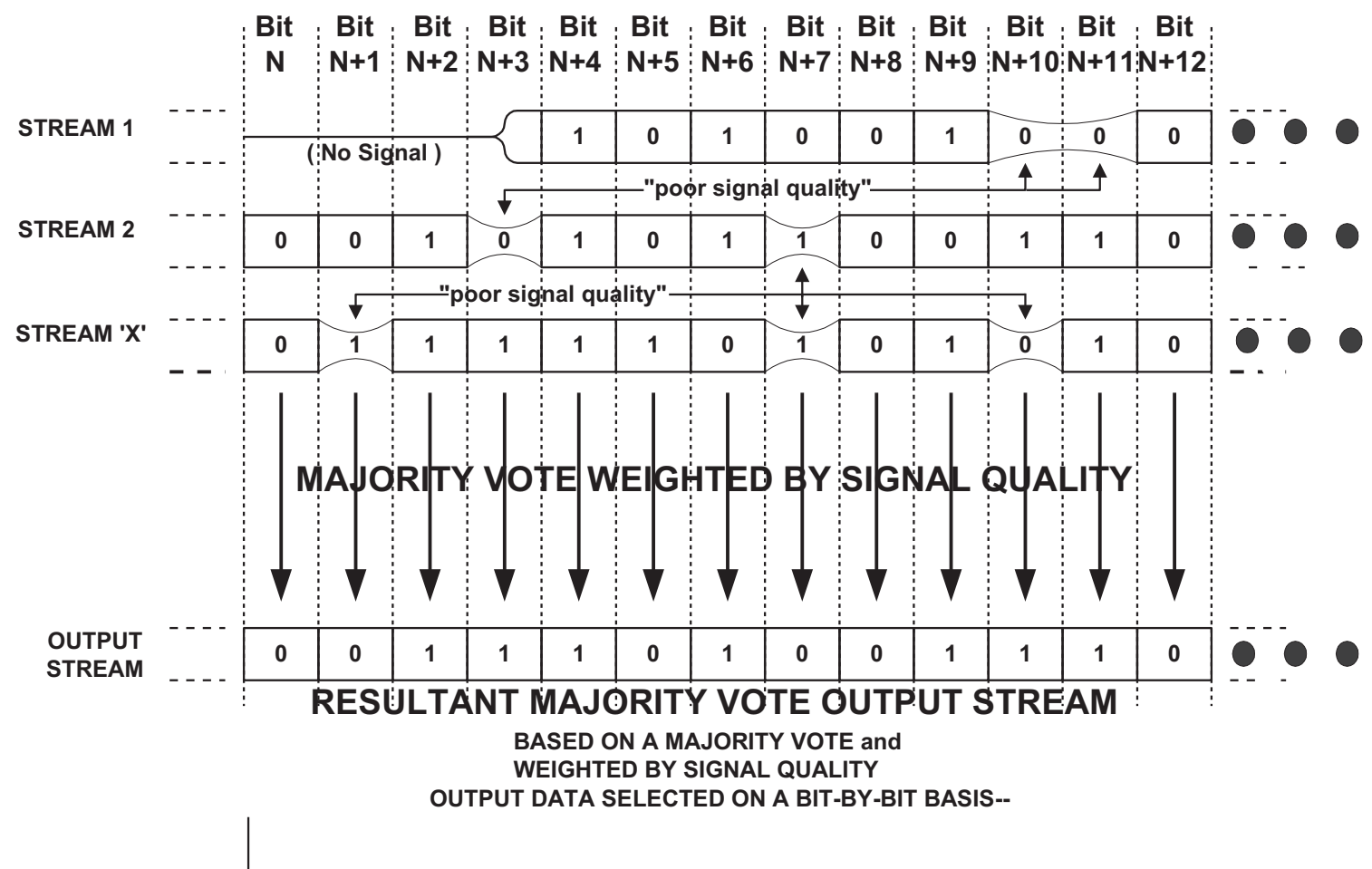

Figure 5 Best Source Selector: Majority Vote Mode (Weighted by Signal Quality)

In the GDP DQE mode, data and quality information is again sent to the CBSS in an encapsulated stream. The encapsulated stream includes a frame sync pattern along with data and interleaved Long Term and Short Term (Bit-by-Bit) data quality. Because the encapsulated stream includes quality data for each bit, the link between the remote site and the CBSS requires a bandwidth of 2.1 times the bit rate. For situations where bandwidth is an issue, there is another GDP encapsulation mode that provides quality for every 4 bits. This mode requires a bandwidth of 1.6 times the bit rate.

\section{Conclusion:}

Best Source Selection has come a long way over the past 25 years. Today's modern Correlating Best Source Selectors (CBSS) are extremely powerful and not only pick the best source but also provide significant performance gains (in some cases over $5 \mathrm{~dB}$ ). CBSS units allow the user to take advantage of spatial diversity with multiple antennas each having a different view angle of the test vehicle. The signals from these antennas can be summed for maximum performance. CBSS units that can simultaneously use a variety of different modes and algorithms, as needed, not only provide performance gains but also provide the best bit-by-bit output solution at any instant in time. Modern units support the data / clock inputs and outputs as well as Ethernet links between the hardware. Correlating Best Source Selectors are here to stay and are at the heart of any new modern test range or launch facility. 